\title{
The Coupled Optoelectronic Problems of Quantum Well Laser Operation
}

\author{
MATT GRUPEN* and KARL HESS \\ Beckman Institute, University of Illinois, 405 N. Mathews Ave, Urbana, IL 61801
}

\begin{abstract}
The treatment of several aspects of quantum well laser simulation are discussed in terms of the Minilase-II simulator. The discussion involves the optical problem and several components of the electronic problem, including bulk transport, carrier scattering into and within the quantum well, and the nonequilibrium LO phonon temperature within the well. Descriptions of these problems are followed by simulation results which show the ways in which they each affect the laser characteristics.
\end{abstract}

Keywords: quantum well, nonlinear gain, spectral hole burning, optical phonons, modulation

\section{INTRODUCTION}

The quantum well laser is a highly complex device containing many interacting physical processes. For example, because a laser requires optical feedback, the output signal depends a great deal on the waveguiding properties of the laser cavity. Also, since the laser is driven by an injected current, classical bulk carrier transport is important to its performance. The optical output is generated when carriers recombine in the quantum well $(\mathrm{QW})$, emitting a photon in the process. As a result, the capture of injected carriers into quantum bound states is critical to laser dynamics. Once captured into a bound state, a carrier can interact with a photon, phonon, or other carriers, and all these interactions are also reflected in the output signal.

Each of the afore mentioned processes has been the focus of much research. However, no single one of them dominates all laser characteristics. Instead, it is important to consider all of these processes, and it is just as important to consider how they couple to and affect one another. Therefore our laser simulator, Minilase-II, has been developed to address all of the physical problems posed by a quantum well laser in such a way that they can be solved self-consistently. This paper will present the simulation methods employed by Minilase-II, including a brief description of the treatment of the electromagnetic and bulk transport problems as well as a more detailed description of carrier dynamics in and around the QW. The paper will also present some simulation results that highlight different aspects of the laser problem.

\section{THE MINILASE-II SIMULATOR}

\section{The Optical Waveguide}

To determine the optical properties of a laser diode, a laser simulator must solve Maxwell's equations for the optical electromagnetic fields in the cavity [1].

* Corresponding author. Tel: (217) 333-9734. Fax: (217) 244-4333. Email: grupen@reg.uiuc.edu 
Minilase-II does this by treating the optical problem in two parts. First the spatial distribution of the optical field is determined by solving the scalar Helmholtz equation [2]

$$
\nabla_{t}^{2} \phi+\left(\varepsilon_{\omega} \omega^{2}-\beta^{2}\right) \phi=0
$$

where $\epsilon_{\omega}$ is the relative permittivity at optical frequency $\omega, c$ is the velocity of light in vacuum, $\phi$ is the optical field, and $\beta$ is the propagation constant. The solution of (1) yields the eigenvector $\phi$, which can be normalized to map photon populations into local photon densities. Minilase-II determines the photon population of each cavity mode by solving a set of photon rate equations

$$
\frac{d S_{\omega}}{d t}=S_{\omega}\left(G_{\omega}-\frac{1}{\tau_{\omega}}\right)+R_{\omega}^{\mathrm{spo}}
$$

where $G_{\omega}$ and $R_{\omega}^{\text {spo }}$ are the gain and the spontaneous emission rate, respectively, of mode $\omega$. The photon lifetime $\tau_{\omega}$ accounts for photon losses at the facets as well as losses due to scattering within the cavity and free carrier absorption.

The gain and spontaneous emission rate of each cavity mode depend on the quantum well band structure, the optical matrix elements, and the occupation probabilities of the lasing states. Minilase-II can treat the band structure and matrix elements as input determined by a separate $\boldsymbol{k} \cdot \boldsymbol{p}$ calculation [3], or, if these are not available, it will use the parabolic band approximation and the Kane model to determine these quantities [4]. The occupation probabilities of the lasing states are functions of the carrier injection into the quantum well, which is determined by the electronic transport models in Minilase-II.

\section{Classical Electronic Transport}

Carrier transport in a QW laser is a multiscale problem that must couple carrier dynamics in the very small quantum regions with the carrier flux in the much larger bulk regions. The carrier fluxes in the bulk regions are treated with classical drift-diffusion theory [5]

$$
j_{n}=-D_{n} \nabla n-\mu_{n} n \nabla E_{c}
$$

where $n$ is the local electron density, $D_{n}$ is the electron diffusion constant, $\mu_{n}$ is the electron mobility, and $E_{c}$ is the conduction band edge. A similar expression is used for holes.

\section{Coupling Classical and Quantum Regions}

The transport in and around the quantum well is more complicated than the bulk transport because classical three-dimensional carriers interact with quantum confined carriers. Because of the small size of the quantum well, incident bulk carriers have a finite probability of traversing the well without scattering into a bound state. Carriers that do scatter into bound states undergo further carrier-carrier, carrier-phonon, and carrier-photon interactions, the dynamics of which are important in determining the output characteristics of the laser. Minilase-II treats these coupled processes according to the models shown schematically in Figures 1 and 2. As Figure 1 shows, the quantum carriers occupying the quasi-continuum states above the well are treated separately from bound quantum carriers within the well. Bulk carriers moving by drift-diffusion are injected ballistically into the quasi-continuum states. The continuum carriers can then transfer ballistically back into the bulk or scatter into bound quantum states by carrier-carrier or carrier-phonon interactions. Within the $\mathrm{QW}$, the carriers are distributed spatially according to drift-diffusion in the lateral direction and their envelope wave functions in the transverse direction. Their distributions in

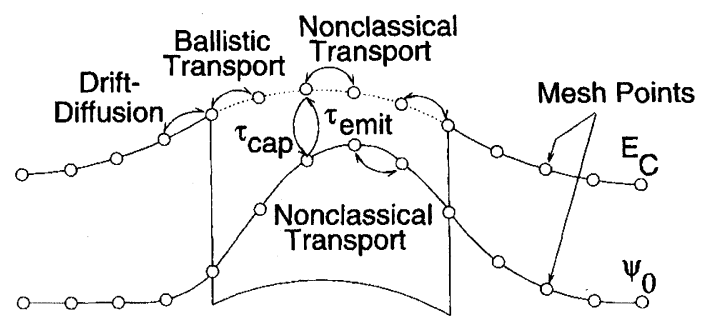

FIGURE $1 \mathrm{~A}$ schematic diagram of the coupling between the classical and quantum regions in the laser as modeled by the MinilaseII simulator 


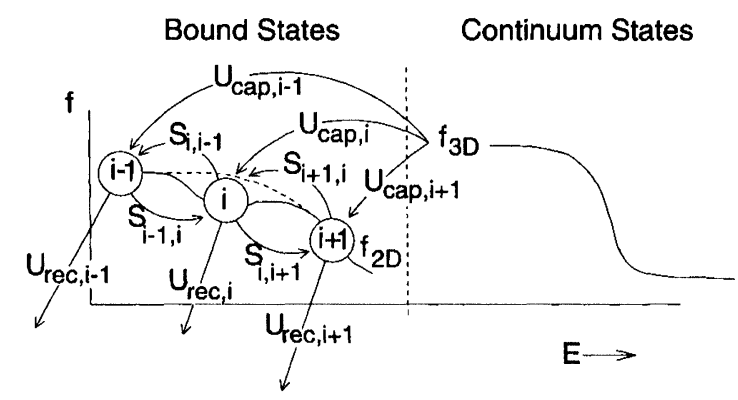

FIGURE 2 A schematic representation of the intrasubband processes in the quantum active region

energy space are calculated by first discretizing the range of bound energies within the well (as shown in Figure 2) and then solving the following equations self-consistently at each energy point.

$$
g_{i} d E_{i} \frac{d f_{i}}{d t}=U_{\mathrm{cap}, i}-U_{\mathrm{rec}, i}+S_{\mathrm{in}, i}-S_{\mathrm{out}, i}
$$

where $g_{i}$ is the density of states at energy point $i, d E_{i}$ is the energy range associated with point $i, f_{i}$ is the occupation probability, $U_{\text {cap }, i}$ is the net capture rate into $i, U_{\mathrm{rec}, i}$ is the net recombination rate, and $S_{\mathrm{in}(\mathrm{out}), i}$ is the net scattering rate into (out of) point $i$ from other surrounding energy points. The net capture and scattering rates are calculated by considering both carrier-carrier and carrier-phonon interactions. Electron-electron interactions are modeled by an artificial mechanism described by the following expressions for capture and intrasubband scattering.

$$
\begin{aligned}
& U_{\mathrm{cap}, i}^{\mathrm{el}-\mathrm{el}}=\left(\int_{E_{c}^{0}}^{\infty} s_{3 \mathrm{D}, i}^{\mathrm{el}-\mathrm{el}} g_{3 \mathrm{D}} f_{3 \mathrm{D}} d E_{3 \mathrm{D}}\right) g_{i} d E_{i} \\
& \times\left[1-f_{i}-f_{i} \exp \left(\frac{E_{i}-F_{n, 3 \mathrm{D}}}{k T_{\mathrm{el}} / q}\right)\right] \\
& S_{\mathrm{in}, i}^{\mathrm{el}-\mathrm{el}}=\sum_{j<i} s_{j, i}^{\mathrm{el}-\mathrm{el}} \exp \left(\frac{E_{j}-E_{i}}{k T_{\mathrm{el}} / q}\right) g_{i} d E_{i} g_{j} d E_{j}\left(1-f_{i}\right) f_{j} \\
& +\sum_{j>i} s_{j, i}^{\mathrm{el}-\mathrm{el}} g_{i} d E_{i} g_{j} d E_{j}\left(1-f_{i}\right) f_{j} \\
& S_{\mathrm{out}, i}^{\mathrm{el}-\mathrm{el}}=\sum_{j<i} s_{i, j}^{\mathrm{el}-\mathrm{el}} g_{i} d E_{i} g_{j} d E_{j}\left(1-f_{j}\right) f_{i} \\
& +\sum_{j>i} s_{i, j}^{\mathrm{el}-\mathrm{el}} \exp \left(\frac{E_{i}-E_{j}}{k T_{\mathrm{el}} / q}\right) g_{i} d E_{i} g_{j} d E_{j}\left(1-f_{j}\right) f_{i}
\end{aligned}
$$

where $E_{c}^{0}$ is the top of the confining energy barrier for the quantum well, $g_{3 \mathrm{D}}$ is the continuum density of states at energy $E_{3 \mathrm{D}}, f_{3 \mathrm{D}}$ is the quasi-Fermi distribution for continuum states with quasi-Fermi level
$F_{n, 3 \mathrm{D}}, f_{i}$ is the occupation probability for bound energy point $i$ with energy $E_{i}$ and density of states $g_{i}$, and $T_{\mathrm{el}}$ is the bound electron temperature. The scattering parameter $s_{3 D, i}^{\mathrm{el}-\mathrm{el}}$ in (5) represents the transition probability of a continuum carrier with energy $E_{3 \mathrm{D}}$ to a bound energy state with energy $E_{i}$. Similarly, $s_{i, j}^{\mathrm{el}-\mathrm{el}}=s_{j, i}^{\mathrm{el}-\mathrm{el}}$ is the scattering parameter for a transition between bound energy points $i$ and $j$. Expressions analogous to (5)-(7) are used to model the holehole interactions.

The electron-phonon equivalents of (5)-(7) are given by the following expressions.

$$
\begin{aligned}
& U_{\text {cap }, i}^{\mathrm{el}-\mathrm{ph}}=s_{3 \mathrm{D}, i}^{\mathrm{el}-\mathrm{ph}} g_{3 \mathrm{D}} d E_{3 \mathrm{D}} g_{i} d E_{i} \\
& {\left[\left(n_{q}+1\right)\left(1-f_{i}\right) f_{3 \mathrm{D}}-n_{q} f_{i}\left(1-f_{3 \mathrm{D}}\right)\right]} \\
& E_{3 \mathrm{D}}=E_{i}+\hbar \omega_{\mathrm{LO}} \\
& S_{\mathrm{in}, i}^{\mathrm{el}-\mathrm{ph}}=\left\{\begin{array}{c}
s_{j, i}^{\mathrm{el}-\mathrm{ph}} n_{q} g_{i} d E_{i} g_{j} d E_{j}\left(1-f_{i}\right) f_{j} \\
\text { for } E_{j}=E_{i}-\hbar \omega_{\mathrm{LO}} \\
s_{j, i}^{\mathrm{el}-\mathrm{ph}}\left(n_{q}+1\right) g_{i} d E_{i} g_{j} d E_{j}\left(1-f_{i}\right) f_{j} \\
\text { for } E_{j}=E_{i}+\hbar \omega_{\mathrm{LO}}
\end{array}\right. \\
& S_{\mathrm{out}, i}^{\mathrm{el}-\mathrm{el}}=\left\{\begin{array}{c}
s_{i, j}^{\mathrm{el}-\mathrm{ph}}\left(n_{q}+1\right) g_{i} d E_{i} g_{j} d E_{j}\left(1-f_{j}\right) f_{i} \\
\text { for } E_{j}=E_{i}-\hbar \omega_{\mathrm{LO}} \\
s_{i, j}^{\mathrm{el}-\mathrm{ph}} n_{q} g_{i} d E_{i} g_{j} d E_{j}\left(1-f_{j}\right) f_{i} \\
\text { for } E_{j}=E_{i}+\hbar \omega_{\mathrm{LO}}
\end{array}\right.
\end{aligned}
$$

where $n_{q}$ is the phonon occupation number given by

$$
n_{q}=\frac{1}{\exp \left(\hbar \omega_{\mathrm{LO}} / k T_{\mathrm{LO}}\right)-1}
$$

where $T_{\mathrm{LO}}$ is the LO phonon temperature. A constant phonon energy $\hbar \omega_{\mathrm{LO}}$ of $36 \mathrm{meV}$ is assumed. All other terms in (8)-(10) have the same meanings as their equivalents in (5)-(7).

\section{The Carrier Temperature}

The electronic problem contains various temperatures corresponding to different systems, e.g. the bound electron temperature $T_{\mathrm{el}}$ in (5)-(7). The temperatures relevant to laser simulation are accounted for with a five-temperature model. The lowest temperature in this model is the ambient temperature or the temperature of the laser's heat sinks, $T_{0}=300 \mathrm{~K}$. The next temperature is associated with the propagation of 
acoustic phonons. The third temperature in the model is associated with the LO phonons. Carriers injected into the quantum well lose energy in part by emitting LO phonons. These phonons remain localized in the well for some time until they decay into two acoustic phonons of approximately opposite $\boldsymbol{q}$-vectors [6], which in turn propagate towards the heat sinks, thus removing heat from the laser. Since the LO phonons have a finite lifetime, they may not be in thermal equilibrium, particularly during strong lasing conditions [7]-[9]. Finally, the fourth and fifth temperatures in this model are the bound electron and bound hole temperatures. In addition to emitting phonons, carriers injected into the well can scatter with other carriers. These carrier-carrier interactions drive the carriers towards a Fermi distribution, however, they do not decrease the total energy of the electron and hole gases. The carrier gases can only lose total energy by emitting LO phonons and gain energy from the hot electrons entering the well. Consequently, as carrier-carrier scattering distributes the energy of the incoming electrons, it raises the average energy of the carrier gas to a temperature $T_{\mathrm{el}(\mathrm{hl})}>T_{\mathrm{LO}}$.

The various temperatures in the five-temperature model are calculated in different ways. The acoustic phonon temperature is calculated by solving a heat flow equation that balances thermal conduction with Joule, recombination, and Thomson/Peltier heat [10]. This part of the temperature problem will be reserved for future publications. For this paper, the acoustic phonon temperature is set to $300 \mathrm{~K}$. The LO phonon temperature is determined by balancing the emission of LO phonons with their decay into acoustic phonons.

$$
\begin{array}{r}
\int_{\left|q_{\min }\right|}^{\left|q_{\max }\right|} D(q) d q \\
+S_{j<i}^{\mathrm{hl}-\mathrm{ph}}-\int_{\left|q_{\min }\right|}^{\left|q_{\max }\right|} D(q) d q \frac{n_{q}-n_{q}^{0}}{\tau_{\mathrm{LO}}^{\mathrm{el}-\mathrm{ph}}}+U_{\mathrm{cap}}^{\mathrm{hl}-\mathrm{ph}}+S_{j<i}^{\mathrm{el}-\mathrm{ph}}
\end{array}
$$

where $\boldsymbol{q}_{\min }$ and $\boldsymbol{q}_{\max }$ determine the range of momentum vectors over which hot $\mathrm{LO}$ phonons interact with free carriers [7, 11], $D(q)$ is the density of LO phonon states, $U_{\text {cap }}^{\text {el(hl) }}-$ ph is the total rate of electron (hole) capture by phonon emission obtained by summing ( 8 ) over all $i, S_{\mathrm{j}<\mathrm{i}}^{\mathrm{el}(\mathrm{hl})-\mathrm{ph}}$ is the net relaxation rate of bound electrons (holes) due to intrasubband phonon scattering obtained from (9) and (10), $n_{q}^{0}$ is the phonon occupation number when $T_{\mathrm{LO}}$ is equal to the acoustic phonon temperature, and $\tau_{\mathrm{LO}}$ is the LO phonon lifetime. Measurements yield a phonon lifetime of approximately 5 ps [12]. Since our model currently neglects carrier energy losses due to electron-hole interactions, we compensate by using a smaller $\tau_{\mathrm{LO}}$ of 1 ps. Finally, $T_{\mathrm{el}(\mathrm{hl})}$ is obtained by iterating between the real space electron (hole) continuity equations, the energy space continuity equation (4) for electrons (holes), and the phonon equation (11) until all are satisfied simultaneously. For the results presented in this paper, both $T_{\mathrm{el}}$ and $T_{\mathrm{hl}}$ are assumed to equal $T_{\mathrm{LO}}$ as determined by (11). This assumption is, of course, not true except in the limits of extremely low or high modulation frequencies. However, at this time the entire five-temperature model is not complete, and therefore we present our current results as an upper limit on LO phonon temperature.

\section{RESULTS}

To illustrate the different coupled problems associated with quantum well lasers, this section will present simulation results that highlight some of the problems discussed in the previous section. Many aspects of optical confinement and bulk carrier transport have been presented and discussed previously (see reference [2]) and will not be repeated here. Instead, let us proceed directly to the explicit treatment of carrier capture into the quantum well active region, the importance of which is demonstrated in Figure 3. This figure compares three experimental modulation responses (discrete points) [13] with those simulated by Minilase-II (continuous lines). As the figure indicates, moving the location of the QW within the separate confinement heterostructure $(\mathrm{SCH})$ significantly affects the modulation response. The different modulation bandwidths are primarily determined by the different optical confinement factors provided by the $\mathrm{SCH}$ regions. The different degrees of low frequency rolloff are determined by the numbers of injected carriers that traverse the $\mathrm{QW}$ without being captured. 


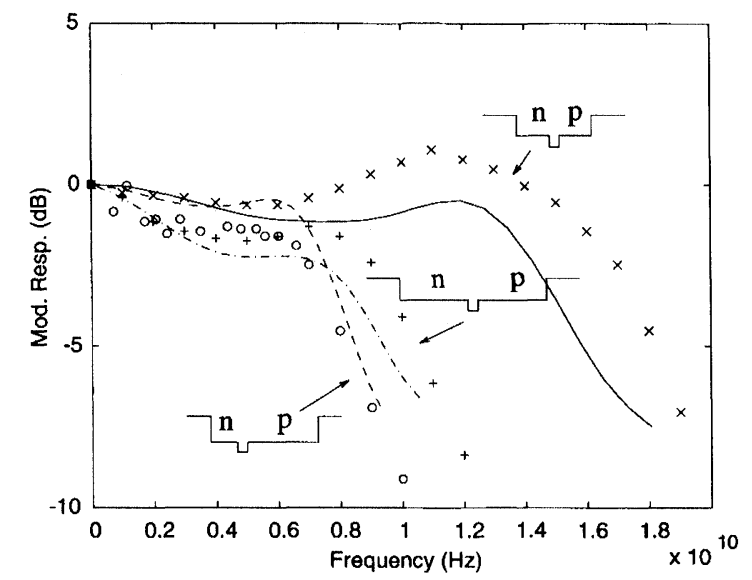

FIGURE 3 The Minilase-II simulation of an experiment in which the position of the QW within the SCH was altered to produce different modulation characteristics. Simulations are continuous lines and measurements are discrete points

These wasted carriers help contribute to the diffusion capacitance of the laser and directly lead to the rolloff of the response in the low frequency range [14]. This connection between wasted free carriers, diffusion capacitance, and the modulation response was not fully understood prior to the explicit treatment of carrier capture by the Minilase-II simulator.

The quantum well capture model used in MinilaseII is also applicable to MQW lasers. Figure 4 shows a set of modulation responses for lasers with different numbers of QW's. As expected, the rate of rolloff in

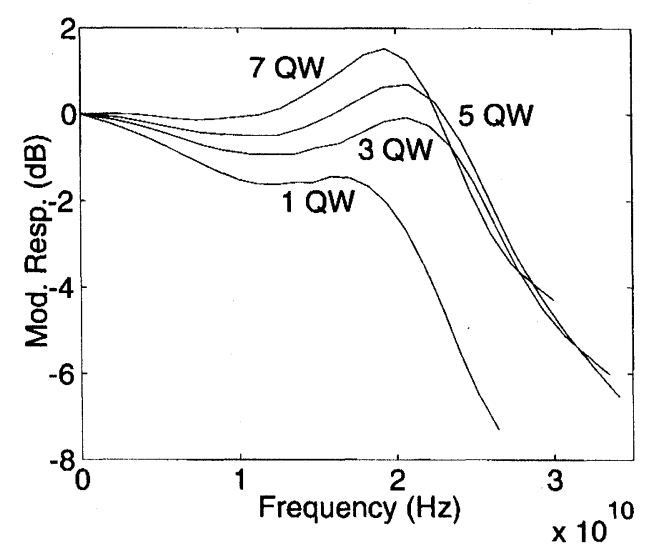

FIGURE 4 A set of modulation responses for different numbers of quantum wells the low frequency range decreases with each additional well because the probability of carrier capture increases. However, note that the frequency of the resonance peak first increases as the number of wells increases to three but then decreases with the further addition of wells. As Figure 5 shows, Minilase-II explains that this behavior is due to a highly nonuniform distribution of electrons and holes in the QW's, i.e. the densities in the wells are not modulated in phase. Consequently, if the number of wells is too large, the less populated wells actually rob from the total differential gain, causing the resonance frequency to decrease. Like the experiment in Figure 3, the result shown in Figure 4 can not be simulated without the explicit treatment of carrier capture.

To illustrate the importance of carrier dynamics in the bound states of the QW, Figure 6 shows a set of modulation responses resulting from different physical assumptions. Each response was calculated for the same bias current of $20 \mathrm{~mA}$, which corresponds to a single facet output power of about $10.35 \mathrm{~mW}$ in each case. However, the response labeled "Fermi" assumes an LO phonon temperature of $300 \mathrm{~K}$ and a quasiFermi distribution for the bound carriers in the quantum well. The response labeled "spectral" also assumes $T_{\mathrm{LO}}=300 \mathrm{~K}$, but a Fermi distribution for bound carriers is not assumed. Instead, this simulation solves (4) self-consistently with the real space transport equations to obtain the bound carrier distribution

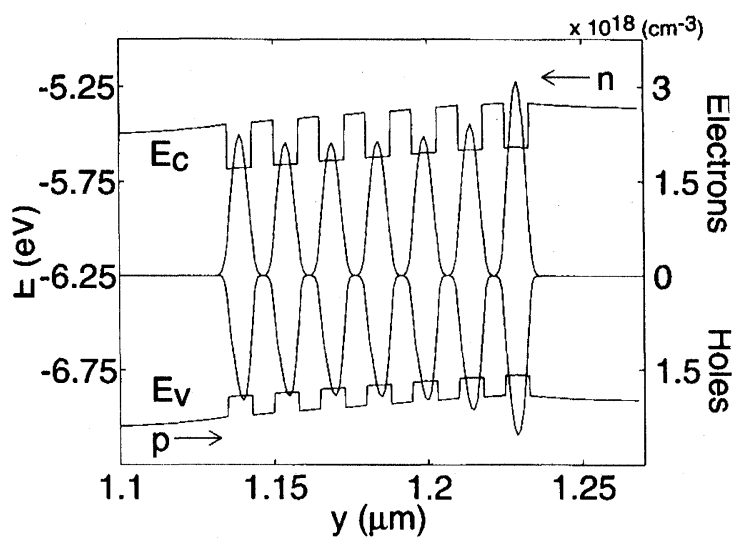

FIGURE 5 Highly nonuniform distributions of bound electrons and holes calculated for an MQW laser 


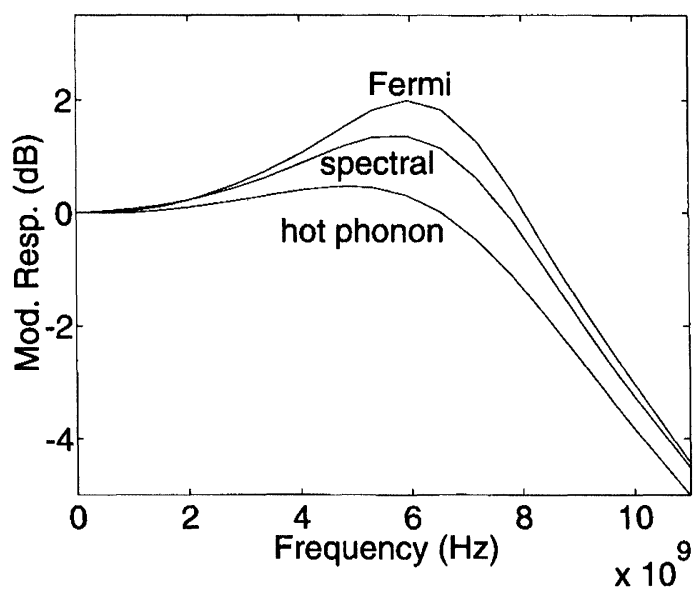

FIGURE 6 Modulation responses calculated under the same bias conditions and different physical assumptions. The response labeled "Fermi" assumes quasi-Fermi distributions for bound carriers and an equilibrium LO phonon temperature of $300 \mathrm{~K}$. The "spectral" response assumes $T_{\mathrm{LO}}=300$, but it accounts for nonFermi carrier distributions. The response labeled "hot phonons" accounts for both elevated phonon temperature and non-Fermi distribution functions

functions and to account for spectral hole burning. Finally, the response labeled "hot phonon" solves both (4) and (11) self-consistently with the real space transport equations to account for the effects of spectral hole burning and nonequilibrium LO phonon temperature.

The gain saturation demonstrated by the "spectral" curve in Figure 6 can be easily understood by referring to Figure 7 . This figure shows the difference between the bound electron distribution function calculated by Minilase-II and a Fermi distribution corresponding to the same electron density. It is clear from the figure that the rapid recombination of lower energy electrons by stimulated emission and the capture of high energy electrons from the continuum states results in a heating of the bound electrons. The decrease in the distribution function near the lasing energy is the reason for the observed gain suppression. Note also from the scale of the figure that the distribution function is nearly Fermi-like. Perturbations of this small magnitude were calculated independently by other groups using sophisticated Monte Carlo calculations [9]. However, the Monte Carlo

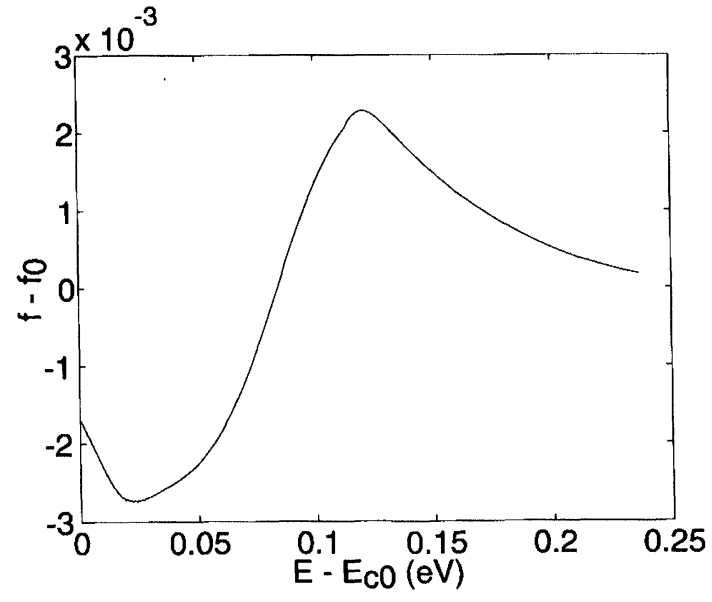

FIGURE 7 The difference between a bound electron distribution function calculated by Minilase-II and a Fermi distribution corresponding to an equal electron density. The $x$-axis shows the bound electron energy with respect to the lowest subband minimum

studies concluded that this change in the distribution function was insignificant, i.e. there is no spectral hole burning. The reason for this conclusion may be that the Monte Carlo method is too cumbersome to simulate an entire laser. Consequently, the optical fields are not fully coupled to the carrier dynamics, and the strength of this coupling is underestimated. An accurate treatment of this effect requires a selfconsistent approach like that used in Minilase-II.

Referring back to Figure 6, the modulation response labeled "hot phonon" shows even more gain suppression than the "spectral" response. In addition to the non-Fermi carrier distributions, the "hot phonon" response accounts for the nonequilibrium LO phonon temperature, which was calculated to be $T_{\mathrm{LO}}$ $\approx 304 \mathrm{~K}$ during $\mathrm{cw}$ operation at a bias of $I \approx 10 I_{\mathrm{th}}$. The elevated phonon temperature causes the bound carrier distributions to spread out to higher energies, causing the differential gain to decrease.

\section{CONCLUSION}

The quantum well laser diode poses several different physical problems, each of which is important in 
determining the output characteristics. The waveguiding properties of the laser cavity determine the near and far field patterns as well as the coupling between the optical field and free carriers in the active region. Classical bulk carrier transport determines current confinement and diffusion capacitance. Also, the behavior of quantum well lasers depends critically on the nonclassical carrier dynamics in and around the well.

While all of these physical processes are critical in determining the output characteristics of the laser, just as critical are the ways in which these different processes couple to one another. With this in mind, the self-consistent laser simulator Minilase-II was developed to address each of these problems in such a way that they could be solved simultaneously, revealing the ways in which each process affects the others and the ways their coupling affects the macroscopic output of the laser. To our knowledge, Minilase-II is the first attempt at fully 2-D self-consistent laser simulation that treats all of the primary carrier transport mechanisms including quantum carrier capture, spectral hole burning, and LO phonon heating.

The Minilase-II simulator reveals several transportrelated effects in quantum well lasers. For example, it clearly demonstrates the relationship between low frequency rolloff in the modulation response and the incomplete capture of quantum carriers and the diffusion capacitance of the device. It also shows that the spatial distribution of electrons and holes in the active region is generally highly nonuniform. Different wells in an MQW laser contain very different densities of carriers, and the densities of electron and holes in a single well are not equal. With regards to spectral hole burning, Minilase-II demonstrates that very slight perturbations in the quantum carrier distribution functions due to stimulated emission are sufficient to produce macroscopically significant gain saturation. Also, the emission of LO phonons by the scattering of bulk carriers into the quantum well and intrasubband scattering of carriers within the well can lead to an elevated carrier temperature that further saturates the differential gain.

\section{Acknowledgements}

We acknowledge support from the NSF through NCCE and the Office of Naval Research.

\section{References}

[1] A.T. Galick, T. Kerkhoven, and U. Ravaioli, "Iterative Solution of the Eigenvalue Problem for a Dielectric Waveguide," IEEE Trans. Micro. Tech., vol. 40, no. 4, pp. 699-705, 1992.

[2] M. Grupen, U. Ravaioli, A. Galick, K. Hess, and T. Kerkhoven, "Coupling the Electronic and Optical Problems in Semiconductor Quantum Well Laser Simulations," in Proc. SPIE OE/LASE Conf., vol. 2146, Los Angeles, CA, 1994, pp. 133-147.

[3] F. Oyafuso, P. van Allmen, M. Grupen, and K. Hess, "Gain Calculation in a Quantum Well Laser Simulator Using an Eight Band $\boldsymbol{k} \cdot \boldsymbol{p}$ Model," VLSI Design, 1996.

[4] E.O. Kane, "Band Structure of Indium Antimonide," J. Phys. Chem. Solids, vol. 1, 1957, p. 249.

[5] K. Hess, Advanced Theory of Semiconductor Devices, Englewood Cliffs, NJ: Prentice Hall, 1988.

[6] G.A. Kosinovsky, "Threshold Current and Modulation Response of Semiconductor Lasers," Ph.D. dissertation, University of Illinois, Urbana-Champaign, 1995.

[7] K. Hess, N. Holonyak, Jr., W.D. Laidig, B.A. Vojak, J.J. Coleman, and P.D. Dapkus, "Hot Electrons and Phonons in Quantum-Well $\mathrm{Al}_{\mathrm{x}} \mathrm{Ga}_{1}{ }^{-} \mathrm{As}$-GaAs Heterostructures," Solid State Comm., vol. 34, 1980, pp. 749-752.

[8] C.-Y. Tsai, Y.-H. Lo, R.M. Spencer, and L.F. Eastman, "A Theoretical Investigation for the Effects of Spectral Hole Burning, Hot Carrier, Hot Phonon, and Carrier DiffusionCapture-Escape on the Limitations of the Modulation Bandwidth in High-Speed Quantum Well Lasers," Digest of IEEE Conf. on Advanced Concepts in High Speed Semiconductor Devices and Circuits, Cornell, Aug. 1995, pp. 77-79.

[9] M.A. Alam and M.S. Lundstrom, "Numerical Studies of Hot Phonon Effects in Quantum Well Lasers," Proc. of Physics and Simulation of Optoelectronic Devices III, eds. M. Osiñski and W.W. Chow, vol. 2399, Feb. 1995, pp. 292-301.

[10] G.H. Song, "Two-Dimensional Simulation of Quantum-Well Lasers Including Energy Transport," Ph.D. dissertation, University of Illinois, Urbana-Champaign, 1990.

[11] L. Rota, M. Grupen, and K. Hess, "Spectral Hole Burning and Carrier-Carrier Interaction in Semiconductor Quantum Well Lasers: A Monte Carlo Investigation," Proc. of Ninth Internat. Conf. on Hot Carriers in Semicond., Chicago, 1995.

[12] J. Shah, Solid-State Electron., vol. 31, 1978, p. 43.

[13] R. Nagarajan, R.P. Mirin, T.E. Reynolds, and J.E. Bowers, "Experimental Evidence of Hole Transport Limited Intensity Modulation Response in Quantum Well Lasers," Electron. Lett., vol. 29, 1993. pp. 1688-1689.

[14] M. Grupen and K. Hess, "Self-Consistent Calculation of the Modulation Response for Quantum Well Laser Diodes," Appl. Phys. Lett., vol. 65, Nov. 1994, pp. 2454-2456. 


\section{Biographies}

Matt Grupen received his B.S. from Penn State University in Engineering Science in 1985. He then attended UCLA where he received an M.S. in Electron Device Physics in 1989. In 1994, he received a $\mathrm{Ph} . \mathrm{D}$. in Computational Electronics at the University of Illinois, where he currently holds a post-doctoral position. e-mail address: grupen@ceg.uiuc.edu

Karl Hess has dedicated the major portion of his research career to the understanding of electronic current flow in semiconductors and semiconductor devices with particular emphasis on effects pertinent to microchip technology. His theories and use of large computer resources are aimed at complex problems with clear application and relevance to miniaturization of electronics. He is currently the Swanlund Professor of Electrical and Computer Engineering, Adjunct Professor for Supercomputing Applications and a Research Professor in the Beckman Institute working on topics related to Molecular and Electronic Nanostructures. He has received numerous awards, for example the IEEE David Sarnoff Field Award for electronics in 1995. e-mail address: k-hess@ux1.cso. uiuc.edu 

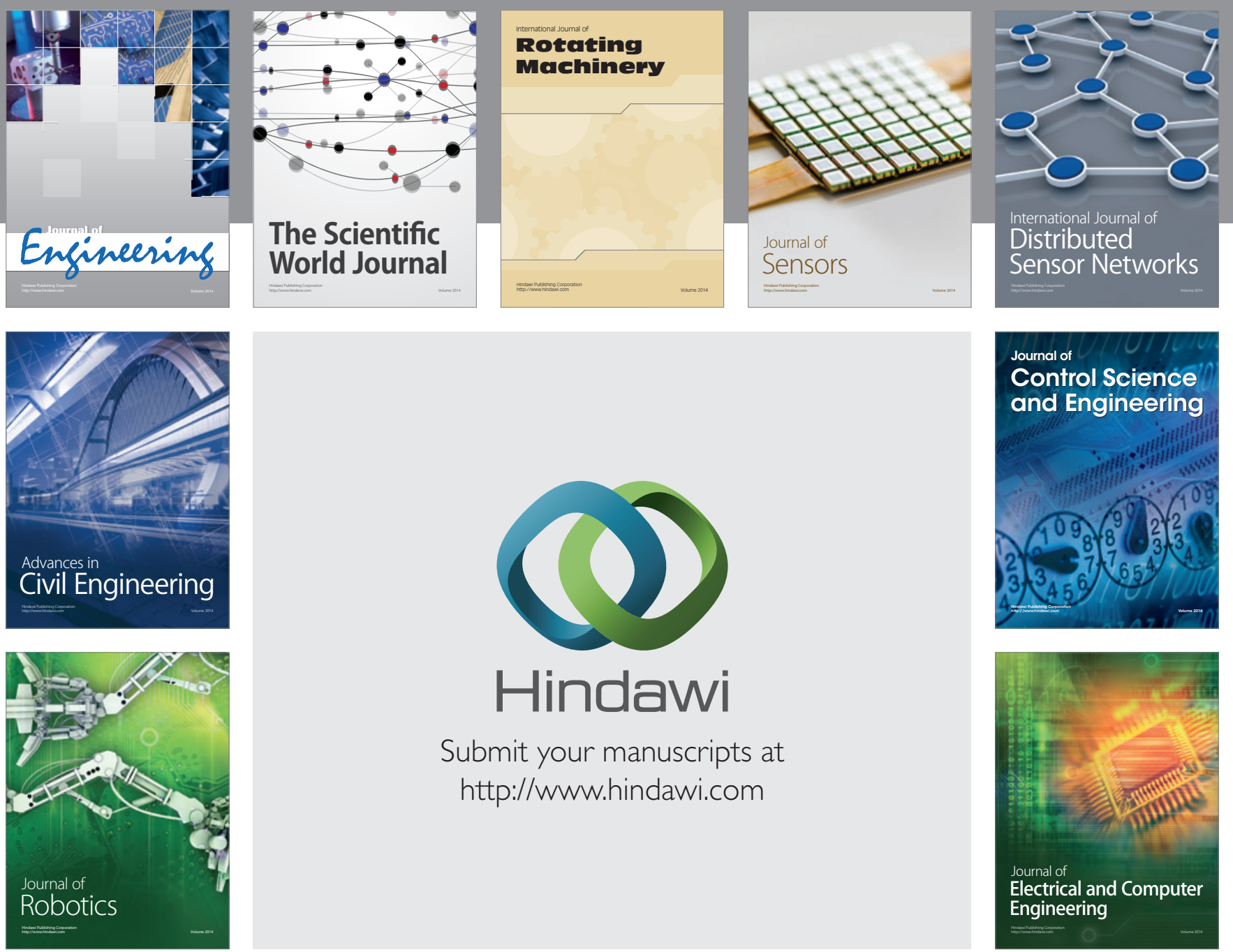

Submit your manuscripts at

http://www.hindawi.com
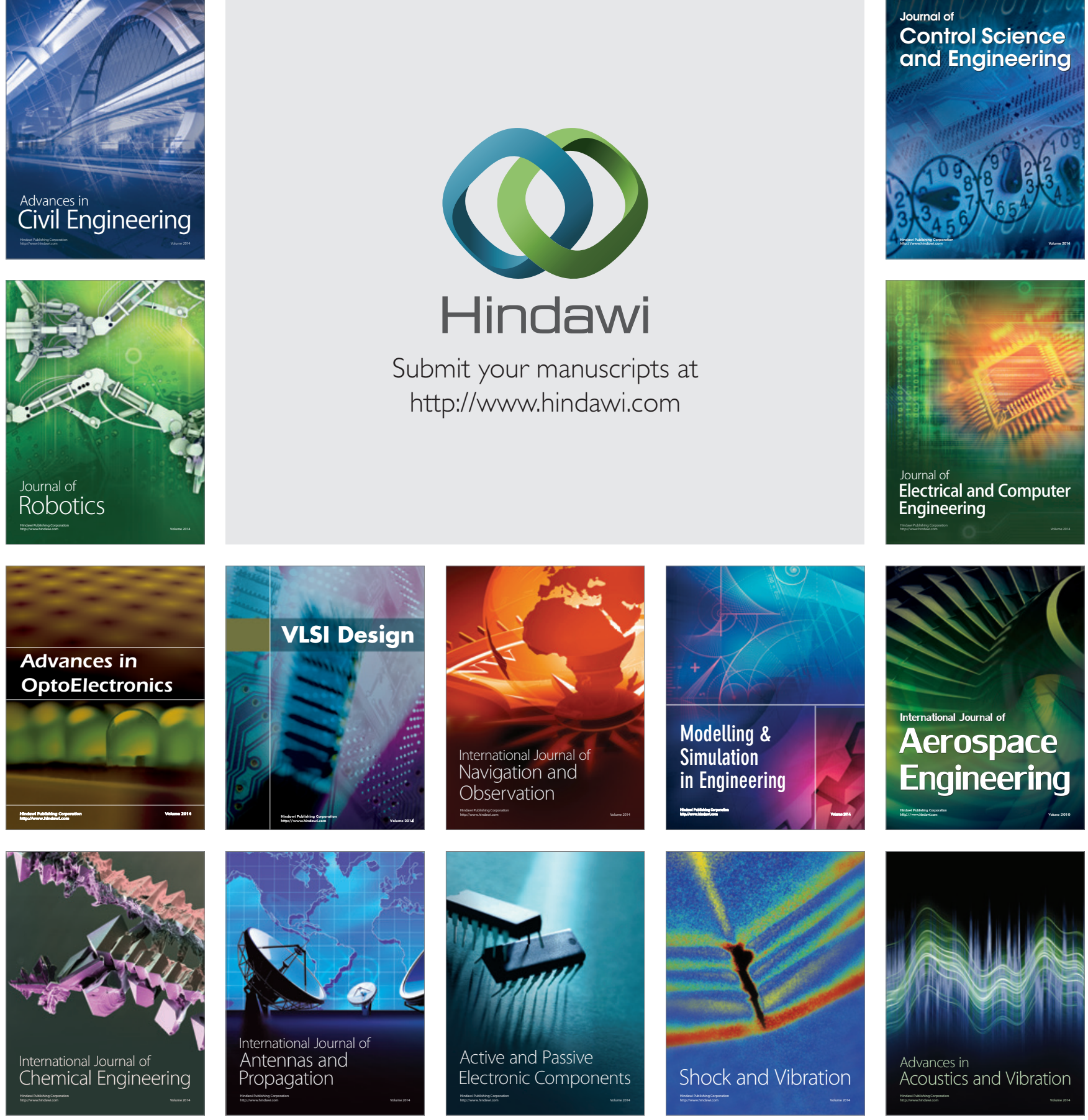